\title{
Hepatitis B Vaccine and Immunoglobulin: Key Concepts
}

\author{
Saibal Das, Kirubakaran Ramakrishnan, Sapan Kumar Behera, Mahalakshmi Ganesapandian, \\ Alphienes Stanley Xavier and Sandhiya Selvarajan*
} Department of Clinical Pharmacology, Jawaharlal Institute of Postgraduate Medical Education and Research (JIPMER),
Puducherry, India

\begin{abstract}
Hepatitis B virus (HBV) immunization is safe and has been accepted worldwide as a routine practice. The target of such vaccination is to induce the immune response in the host, resulting in the prevention of replication of HBV. There are several immunological and clinical factors which determine the clinical efficacy and safety of the HBV vaccine. In this article we have highlighted the response of the host immune system to HBV vaccination (immunogenicity), efficacy, and safety of the vaccine, issues with booster dosing, paths of development (preclinical and clinical) of the HBV vaccine, novel and upcoming strategies for improvement of HBV vaccination, and the concept of therapeutic HBV vaccination. The different aspects and regulatory recommendations pertaining to HBV vaccine development are also discussed. The new strategies for improvement of HBV vaccination include pre-S1 and pre-S2 portions of the HBV surface antigen, increasing the antigen dose, accelerated vaccination schedules, alternative vaccination route, use of adjuvants like immunostimulatory DNA sequences, etc. Therapeutic vaccination is being explored for initiation of a multifunctional and multispecific T cell response against the major HBV antigens and also effective activation of humoral immunity for viral control.
\end{abstract}

Citation of this article: Das S, Ramakrishnan K, Behera SK, Ganesapandian M, Xavier AS, Selvarajan S. Hepatitis B vaccine and immunoglobulin: Key concepts. J Clin Transl Hepatol 2019;7(2):165-171. doi: 10.14218/JCTH.2018.00037.

\section{Introduction}

Human beings are the sole major reservoir of hepatitis $B$ virus (HBV) and hence a complete control strategy by HBV vaccination could lead to virus eradication. ${ }^{1}$ Despite major development and advances in antiviral therapy, primary prevention of infection by vaccination is of utmost importance in public health. ${ }^{2}$ Global vaccination is, in fact, the most economical method employed to reduce the problem of HBV infection. ${ }^{3}$

Keywords: Antibody; Development; Immune response; Immunoglobulin; Vaccine. Abbreviations: anti-HBCAg, anti-core HBV antigen; anti-HBs, anti-HBV surface antibody; cccDNA, covalently closed circular DNA; HBeAg, hepatitis B e antigen; HBIG, hepatitis B immune globulin; HBsAg, HBV surface antigen; HBV, hepatitis B virus; WHO, World Health Organization.

Received: 11 June 2018; Revised: 16 November 2018; Accepted: 11 May 2019

*Correspondence to: Dr. Sandhiya Selvarajan, Department of Clinical Pharmacology, Jawaharlal Institute of Postgraduate Medical Education and Research (JIPMER), Puducherry 605006, India. Tel: +91-9443492922, E-mail: sandhiyaselvarajan@ gmail.com
The goal of active immunization against HBV is to boost the immunity in the host resulting in loss of HBV surface antigen (HBsAg) and continued control of HBV replication. Vaccination strategies against HBV include administration of traditional HBsAg vaccine, human anti-HBV surface antibody (anti-HBs), T cell vaccine, DNA vaccines, apoptotic cells expressing HBV antigens, and viral vectors expressing HBV proteins. ${ }^{4}$ Parenteral HBV immunoglobulin is occasionally used to provide instant protection until an effective response in the host immune system occurs and also among individuals who do not form an effective immune response to conventional HBV vaccination. ${ }^{5}$

In 1991, the World Health Organization (WHO) endorsed that all the countries should integrate $\mathrm{HBV}$ vaccination in their national immunization programs, ${ }^{3}$ and this vaccine should be given on day 0 and at the end of 1 month and 6 months. ${ }^{6}$ Infant immunization is considered an effective strategy to prevent HBV infection and this has been incorporated in the national immunization programs of most of the countries. ${ }^{7}$ However, catch-up strategies, adult vaccination and dealing with special populations are also important. ${ }^{8}$

With regard to HBV protection, both monovalent and combined vaccines were found to provide similar seroprotection or vaccine response rates. ${ }^{9,10}$ HBV vaccines are available as a single-antigen formulation and in combination with other vaccines. The single antigen vaccines are recommended for use at birth. The combined vaccines are usually not recommended at birth ('Pediarix' for individuals aged 6 weeks- 6 years and 'Twinrix' for individuals aged $\geq 18$ years). ${ }^{11}$ The recommended doses of hepatitis $B$ vaccine, by group and vaccine type, is enumerated in Table $1 .{ }^{11}$ The schematic representation of the mechanism of action of $\mathrm{HBV}$ vaccine is depicted in Fig. 1.

\section{A brief history of the HBV vaccine}

The first HBV vaccine (a heat-treated form of HBV) was developed by Blumberg and Millman ${ }^{12}$ in 1969. The United States Food and Drug Administration approved a plasmaderived HBV vaccine produced by Merck Pharmaceuticals in 1981 that involved inactivation of viral particles in the blood which had been collected from HBsAg-positive donors. In 1986, the subsequent generation of genetically engineered (or DNA recombinant)-a highly purified HBV vaccine-was synthetically prepared without containing any of the blood products. ${ }^{13}$ In the present time, all recombinant vaccines which contain HBsAg are expressed in yeast Saccharomyces cerevisiae, Hansenula polymorpha, Pichia pastoris or mammalian (Chinese hamster ovary) cells. ${ }^{14}$ 
Das S. et al: HBV vaccine and immunoglobulin: Key concepts

Table 1. Recommended doses of hepatitis B vaccine, by group and vaccine type

\begin{tabular}{|c|c|c|c|c|c|c|c|c|c|}
\hline \multirow[b]{3}{*}{ Population } & \multirow[b]{3}{*}{$\begin{array}{l}\text { Age } \\
\text { (years) }\end{array}$} & \multicolumn{4}{|c|}{ Single-antigen vaccine } & \multicolumn{4}{|c|}{ Combination vaccine } \\
\hline & & \multicolumn{2}{|c|}{ 'Recombivax' } & \multicolumn{2}{|c|}{ 'Engerix' } & \multicolumn{2}{|c|}{ 'Pediarix'” } & \multicolumn{2}{|c|}{ 'Twinrix' } \\
\hline & & $\begin{array}{l}\text { Dose } \\
(\mu \mathrm{g})\end{array}$ & $\begin{array}{l}\text { Volume } \\
(\mathrm{mL})\end{array}$ & $\begin{array}{l}\text { Dose } \\
(\mu \mathrm{g})\end{array}$ & $\begin{array}{l}\text { Volume } \\
(\mathrm{mL})\end{array}$ & $\begin{array}{l}\text { Dose } \\
(\mu \mathrm{g})\end{array}$ & $\begin{array}{l}\text { Volume } \\
(\mathrm{mL})\end{array}$ & $\begin{array}{l}\text { Dose } \\
(\mu \mathrm{g})\end{array}$ & $\begin{array}{l}\text { Volume } \\
(\mathrm{mL})\end{array}$ \\
\hline \multirow{4}{*}{$\begin{array}{l}\text { Normal } \\
\text { individuals }\end{array}$} & Birth-10 & 5 & 0.5 & 10 & 0.5 & $10^{*}$ & 0.5 & NA & NA \\
\hline & $11-15$ & $10^{\$}$ & 1 & NA & NA & NA & NA & NA & NA \\
\hline & $11-19$ & 5 & 0.5 & 10 & 0.5 & NA & NA & NA & NA \\
\hline & $\geq 20$ & 10 & 1 & 20 & 1 & NA & NA & $20^{\dagger}$ & 1 \\
\hline \multirow{2}{*}{$\begin{array}{l}\text { Hemodialysis patients } \\
\text { and other immune- } \\
\text { compromised persons }\end{array}$} & $<20$ & 5 & 0.5 & 10 & 0.5 & NA & NA & NA & NA \\
\hline & $\geq 20$ & 40 & 1 & 40 & 2 & NA & NA & NA & NA \\
\hline
\end{tabular}

Abbreviation: NA, not applicable.

${ }^{*}$ 'Pediarix' is approved for use in persons aged 6 weeks through 6 years (prior to the $7^{\text {th }}$ birthday).

' 'Twinrix' is approved for use in persons aged $\geq 18$ years.

${ }^{\S}$ Adult formulation administered on a two-dose schedule.

\section{Adequate response after $\mathrm{HBV}$ vaccination}

Several possible questions can be raised concerning the characteristics of anti-HBs antibodies: ${ }^{15}$ A) Is there a titer effect (higher antibody titer affords greater protection)? B) Is there a delay in the antibody response? C) Is the antibody persistent or boosters required? D) Does the vaccinestimulated anti-HBs offer the same protection as naturally arising anti-HBs?

Several clinical trials have been performed to investigate the most optimal and effective vaccine dose and schedule of

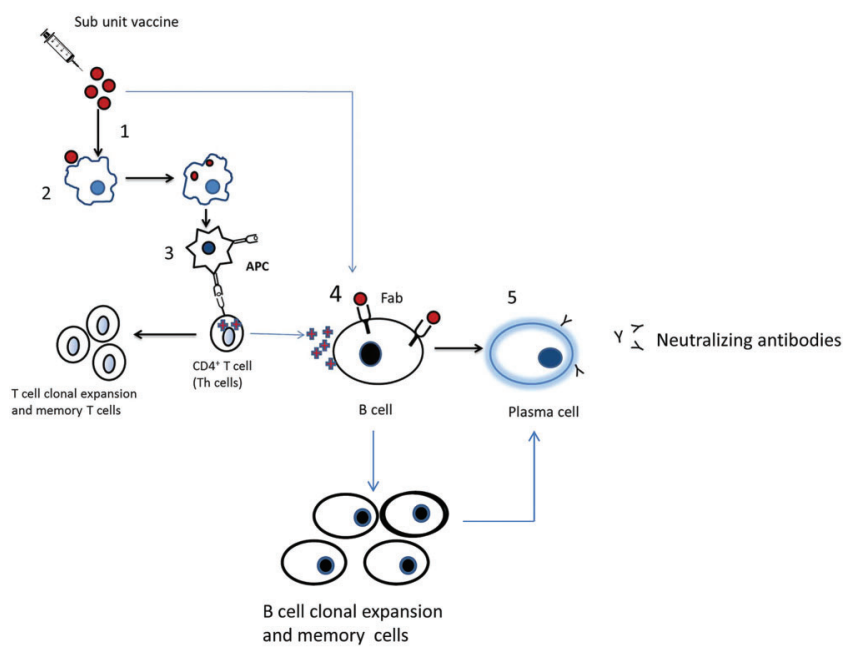

Fig. 1. Schematic representation of the mechanism of action of HBV vaccine. 1 . The injected $H B V$ vaccine containing $H B s A g$ proteins are engulfed and processed by the antigen presenting cells. 2 . The antigen presenting cells process the antigen and attach the same to the surface of the antigen presenting cells. 3 . The antigen presenting cells present the antigen to the $T$ helper cells, leading to clonal expansion of the T cells as well as production of memory T cells. 4 . The antigen can be recognized directly by $B$ cells, producing a weak immune response, with binding of the antigen to the Fab region on the $B$ cell receptor and secondary signaling from cytokines released by T-helper cells; B cells begin somatic hypermutation at the Fab region, which further increases the corresponding fit between the Fab region and the antigen. 5 . The $B$ cells mature to plasma cells to produce neutralizing antibodies. They also undergo clonal expansion and memory cell formation for future defense. vaccination in different subject groups like adults, infants and neonates, and immune-suppressed patients. ${ }^{16}$ The ideal vaccine will produce sufficient titer with minimal delay, remain persistent and offer the same protection as naturally acquired anti-HBs. ${ }^{17}$ The antibody titer after vaccine administration ranges from $<10 \mathrm{IU} / \mathrm{L}$ (non-responder) to $>10000 \mathrm{IU} / \mathrm{L} .{ }^{18} \mathrm{At}$ least three doses are necessary for a minimally acceptable immune response (anti-HBs antibody titer $\geq 10 \mathrm{IU} / \mathrm{L}$ estimated 1-2 months after administering the last dose of the vaccine). ${ }^{19}$ The anti-HBs titer declines very fast, within 12 months, and comparatively slowly thereafter. ${ }^{16}$ Advanced models with mathematical algorithms can predict such a declining trend. ${ }^{20}$

\section{HBV vaccination}

\section{Healthy individuals}

In healthy individuals, the HBV vaccine is given at baseline, 1 month and 6 months. A booster dose may not be needed in apparently healthy individuals.

\section{Liver transplanted patients}

For liver transplanted patients, the classic schedule is 0,1 month, 6 months and booster dose in the subsequent 1 or 2 years based on serology is recommended. ${ }^{21}$ In liver transplant candidates, the rate of seroconversion is significantly low compared to healthy individuals. The low rate of seroconversion can be attributed to disease severity as well as immunosuppressant medication use. To overcome vaccine failure approaches like accelerated schedule, increased dose and repeated vaccinations have been tried with improved success. ${ }^{22-24}$ Earlier individuals undergoing liver transplant for chronic hepatitis $B$ infection were solely dependent on antivirals and/or hepatitis $B$ immune globulin (HBIG), but newer vaccines have been developed to reduce the use of HBIG and also with an aim for better protection against reactivation.

\section{Poor response to $H B V$ vaccination}

Unresponsiveness to HBV vaccination is considered as a serum anti-HBs titer $<10 \mathrm{IU} / \mathrm{L}$ after an initial course of 
vaccination. ${ }^{25}$ A flaw in the $T$ cell compartment-specific for HBsAg is the principal factor. ${ }^{16}$ Hindering effect on anti-HBs response is caused by old age, smoking, body mass index, gender, concomitant chronic disease, ${ }^{16}$ gluteal vaccination, hemodialysis, immunodeficiency, ${ }^{15,26}$ chronic HBV infection, celiac disease, inappropriate storage conditions, etc. ${ }^{27}$ Cirrhotic patients might be at higher risk for flares from immunemediated hepatitis after HBV vaccination. ${ }^{4}$ The absence of a satisfactory response may be due also to genetic predispositions linked to the major histocompatibility complex. Considering the other factors, a meta-analysis has shown that there was no statistical correlation between alcoholism or different vaccination schedules and response to HBV vaccination. ${ }^{26}$

\section{HBsAg mutants}

There are numerous reports on vaccine failure due to HBV mutants, ${ }^{16}$ although the prevalence of such mutants is uncertain. Most of the antibodies which appear after the natural course of infection as well as vaccination are directed towards the determinant epitope cluster which is located in the major hydrophilic domain of HBsAg. Some mutants may also arise in the regions targeted by vaccine and thus affect HBsAg antigenicity. The effect of such HBsAg mutants on public health vaccination programs are governed by their prevalence, infectivity, pathogenicity, and cross-immunity. ${ }^{16}$

These mutations are mostly due to single point mutations in the $\alpha$ determinant of the surface antigen ( $\mathrm{S}$ gene mutations), which is the target site of neutralizing antibodies. Ultimately these antibodies cannot bind to these mutants. These mutants are of prime concerns as the vaccinated people are affected by these mutants. ${ }^{28}$

Available evidence suggests that mutants are selected by vaccines. A survey of hepatitis $B$ surface variant infection in children conducted in Taiwan shows that the prevalence of vaccine escape mutant significantly raised from the prevaccination to postvaccination era. The prevalence was $7.8 \%$ in 1984 , which increased to $19.6 \%$ in 1989 and $23.1 \%$ in 1999. The prevalence was higher in the vaccinated children group $(32 \%)$ compared to the unvaccinated group $(9 \%) .{ }^{29}$ Another study in an Italian population found the prevalence of G145R mutation to be $3.1 \%$ among 256 patients studied from 2007$2011 .{ }^{30}$ In China, it has been observed that post mass vaccination in children the prevalence of escape mutants in children increased from $6.5 \%$ in 1992 to $14.8 \%$ in 2005, with the predominant mutation being G145R. ${ }^{31}$

\section{Long-term efficacy of HBV vaccines}

There are many issues regarding the duration of protection by the HBV vaccine. Most studies concerned with long-term protection were conducted with the plasma-derived vaccine and not with the recombinant one. Although antigen content of both these vaccines is the same, some physical and chemical properties may vary. ${ }^{15,17}$ Between $8-42 \%$ of the people with protective antibody following vaccination lost it within 5 years. However, the time taken for the disappearance of antibody shows wide variation. ${ }^{32}$ Although the possibility of developing HBsAg among subjects who respond to an HBV vaccine is almost nil, risks persist for developing anti-core $\mathrm{HBV}$ antigen (anti-HBcAg) conversion with the decline of anti-HBsAg titer. However, it was demonstrated that after a usual three-dose HBV vaccination, approximately $90 \%$ (range: $74-100 \%$ ) of the subjects who received the vaccine remained protected for $\geq 30$ years regardless of the anti-HBs antibody titer. $^{33}$

\section{Booster dosing}

For many years, booster immunizations were advocated for individuals having increased risk of HBV infection (mostly occupational), when the anti-HBs titer reaches the minimal protective titer $\geq 10 \mathrm{IU} / \mathrm{L}$ (in most countries) or $100 \mathrm{IU} / \mathrm{L}$ (in the UK). ${ }^{16}$ Generally, anti-HBS titer above $10 \mathrm{mIU} / \mathrm{mL}$ is regarded as protective. Some countries have kept anti-HBs levels of $100 \mathrm{mIU} / \mathrm{mL}$ as protective, to provide greater confidence that a specific response has been established. Some assays are also not specific at lower levels. ${ }^{34}$

Currently, many national authorities no longer advocate regular boosters but recommend the serological control 1-3 months after termination of the HBV vaccination schedule. ${ }^{16}$ In a statement by the European Consensus Group on HBV Immunity, a list of recommendation was laid. ${ }^{35}$ Booster is not mandated for immunocompetent subjects responding effectively to the initial vaccination course. Periodic testing of anti-HBs antibodies and booster injection is needed for immune-compromised patients when the titer is $<10 \mathrm{IU} / \mathrm{L}$. Longstanding monitoring should check the non-appearance of breakthrough episodes to detect carrier state after $>15$ years. Additional doses need to be given to those individuals who inadequately responded to the primary vaccination course. Booster doses can be tried in cases of breakthrough infections.

\section{Safety of HBV vaccines}

HBV vaccines are mostly safe. However, absolute contraindication to HBV vaccination is hypersensitivity to yeast or any vaccine constituent. In addition, anaphylaxis, deranged liver enzymes, erythema multiforme, arthritis, multiple sclerosis, Guillain-Barré syndrome, neuritis, thrombocytopenia, optic neuritis, transverse myelitis, and alopecia have been reported. Few reports of arthritic, neurological, gastrointestinal and immunological adverse reactions subsequent to HBV vaccination are available. ${ }^{36}$ However, surveillance studies conducted in the USA have demonstrated that there was no association between serious adverse events and HBV vaccination. The Centers for Disease Control and Prevention has ruled out any confirmed evidence that HBV vaccine causes chronic illness, including multiple sclerosis, rheumatoid arthritis, chronic fatigue syndrome, or autoimmune disorders. ${ }^{36}$ The Global Advisory Committee on Vaccine Safety has also confirmed that HBV vaccination is very safe. ${ }^{37}$

\section{Adjuvants in recombinant HBV vaccines}

Modern recombinant vaccines are very refined and contain less antigenic components (lesser immunogenicity). As a result, adding adjuvants is essential to induce a better immune response. Among the adjuvants, aluminum salts are widely used. These salts can form insoluble particles, cause retention and release of vaccine antigens gradually like a depot, and thereby induce innate immunity. ${ }^{14}$ The various adjuvant systems used with recombinant HBV vaccines are AS01B (liposomal), AS01E (liposomal), AS02A (oil in water emulsion), AS02B (oil in water emulsion), AS02V (oil in water emulsion), AS03 ( $\alpha$-tocopherol and squalene in oil in water emulsion), AS04 (0.5 mg/dose of aluminum phosphate/hydroxide in sodium chloride and water), etc. ${ }^{14}$ 
Das S. et al: HBV vaccine and immunoglobulin: Key concepts

\section{Preclinical development of HBV vaccine ${ }^{38}$}

The different preclinical models for HBV vaccine evaluation includes cell lines (e.g. HepG2, Huh7 HepAD38, HepaRG) which are useful for replication and transcription studies. These can be cultured indefinitely. However, this model can elicit distorted and inadequately functional innate immune responses and results in loss of liver architecture. Primary human hepatocytes are also useful to study HBV replication and transcription, although these cannot be cultured indefinitely. Distortion of liver architecture is also a drawback in this model. The duck model is an ortholog model and is useful to study the life cycle of the virus and drug metabolism. Chronic infection and covalently closed circular DNA (referred to as cccDNA) formation can be studied. However, there is a lack of proper research tools to use this model. The tupaia (treeshrew) model is also useful to study HBV infectivity and viral lifecycle. However, proper research tools for use of this model are lacking. The woodchuck (groundhog) model is also an ortholog model and is useful for studying infection of HBV and viral lifecycle, cccDNA formation, drug metabolism, and carcinogenicity. There is also a lack of good tools to cultivate this model. The mouse is not an effective model to study HBV infectivity, yet this model can still be utilized for immunotherapy and drug metabolism studies. The chimpanzee is an ideal model for studying experimental HBV infection, viral lifecycle, immune responses, and drug metabolism as well as to perform vaccine research. However, the disease is less severe in this species than in humans. Ethical concerns also prevail using this model. The WHO guideline is available for preclinical assessment of an HBV vaccine. ${ }^{39}$ As confirmatory studies with recombinant $\mathrm{HBsAg}$ vaccines have been performed already in the chimpanzee model, extensive preclinical studies are no longer required for $\mathrm{HBsAg}$ protein-based new vaccines.

\section{Clinical development of the HBV vaccine}

The HBV vaccine trials are carried out in individuals who are anti-HB or HBsAg negative. ${ }^{17}$ An ideal population to study an $\mathrm{HBV}$ vaccine is the one with a higher risk of infection that can be attributed mainly to environmental circumstances. Further, the infected individuals should develop and retain the antigen for a sufficient time. HBsAg detection could help to identify any infection and the subjects would not develop anti-HB without producing HBsAg first. ${ }^{17}$ An HBV vaccine developmental plan should consider the target population and the sociocultural aspects, the risk for the target disease and vaccine, the incidence of HBV infection and related environmental factors, the proper dose and route of administration, induction of herd immunity, and regulatory requirements. ${ }^{40}$

\section{Sample size calculation}

The detailed calculation of sample size in conducting a classical HBV vaccine trial has been described by Lustbader et $a .{ }^{17}$ The infection rate escalates as the months of exposure increases. Therefore, the required sample size is a decreasing function of time. Assuming constant error probabilities of 0.01 , the sample sizes required for $2,3,4,5,6,7,8,9,10$, 11 and 12 months of exposure are $355,167,106,60,59,51$, $42,33,32,27$ and 24 respectively in each group. It is unlikely that there would ever be all the volunteers available at one time to initiate the trial and hence a staggered start design might be required. ${ }^{17}$
Regulatory recommendations for clinical development of HBV immunoglobulins

WHO mentions the use of HBV immunoglobulin in newborn infants whose mothers are HBsAg-positive, in anyone following exposure to the percutaneous or mucous membrane with HBsAg-positive blood or body fluids, following sexual exposure to an HBsAg-positive person, or to protect from recurrent $\mathrm{HBV}$ infection following liver transplantation. The HBV immunoglobulins are generally considered as adjuvants to the vaccine. ${ }^{41} \mathrm{~A}$ recent study showed a better response rate in HBV perinatal transmission in the group who received both immunoglobulin and $\mathrm{HBV}$ vaccine. ${ }^{42}$

The European Medicines Agency has published its guidelines on the clinical development of HBV immunoglobulins. ${ }^{43}$ The European Medicines Agency recommends batch-to-batch consistency, after a manufacturing process change, such that it has to be demonstrated that the change has not compromised the quality, efficacy, and safety of the new vaccine. The pharmacokinetic profile ought to be tested in $\geq 20$ adult healthy volunteers who are negative for HBsAg, anti-HBs, and anti-HBc. A pharmacokinetic study in children is not mandatory because data from adults can be mathematically extrapolated to children. The dose should be consistent with the current regulations on human plasma-derived HBV immunoglobulin. If the dose varies, supporting clinical data is needed. For efficacy evaluation, the sample size should be adequate to provide confirmatory data and the recommended baseline data include disease status (acute or chronic HBV), presence of any coinfection with other viral infections (e.g. hepatitis C, D, and human immunodeficiency virus), use of any exposure immunosuppressive agents and antiviral drugs, time elapsed between liver transplant and start of prophylaxis, and circulating HBV DNA titer.

If HBV immunoglobulin is developed as a monotherapy for a particular indication then the anti-HBs titers should be maintained at $>500 \mathrm{IU} / \mathrm{L}$ and $>100-150 \mathrm{IU} / \mathrm{L}$, and for patients with and without active viral replication respectively. For HBV immunoglobulin together with antiviral therapy, targets for trough titers of HBV immunoglobulin should be justified. Intersubject variability of anti-HBs and use of antivirals should be reported. The primary end-point should be the proportion of patients showing recurrence to HBV infection as demonstrated by positive results for $\mathrm{HBsAg}$ and/ or hepatitis $B$ e antigen ( $\mathrm{HBeAg}$ ). Secondary end-points will include anti-HBs titer, circulating HBV DNA titer, time to recurrence of HBV infection, and overall survival. Supportive evidence of efficacy can include liver histopathology reports. A pretreatment serum sample from each subject should be stored at $-70^{\circ} \mathrm{C}$ for future tests. All the end-points need to be estimated prior to HBV immunoglobulin administration and then periodically for $\geq 12$ months. It is recommended to accumulate long-term data on recurrence and overall survival with either a risk management plan or a postapproval efficacy study. All adverse events need to be recorded for analysis and reporting. The effect of passive transmission of hemagglutinins (anti-A/anti-B) should be assessed in patients who have received high doses of HBV immunoglobulin. Data in the elderly population are not needed, as the benefit/risk may be extrapolated from adult data. 


\section{HBIG in liver transplantation}

HBIG is indicated to prevent reinfection in individuals who are undergoing liver transplantation due to hepatitis. If the HBIG is developed with indication for prevention of hepatitis $B$ recurrence following liver transplantation then the clinical development to evaluate efficacy should be done in patients who have undergone liver transplant for liver failure caused by hepatitis B. In the same setting, additional data like antigen-driven complement fixation, opsonisation, phagocytosis, antibody-dependent cell mediated cytotoxicity may also be submitted. The new immunoglobulin for the abovesaid indication should be studied with at least 25 participants and also with the intended mode and route for administration. The European Medicines Agency accepts open-label uncontrolled studies for clinical development and also recommends to use end-points measuring the proportion of patients who develop a recurrence of hepatitis $B$ as demonstrated by positive results for $\mathrm{HBsAg}$ and/or $\mathrm{HBeAg}$, titer of anti-HBs, titer of circulating hepatitis $B$ virus DNA, time to recurrence of hepatitis $B$, and overall survival. ${ }^{43}$

\section{Regulatory recommendations for clinical development} of recombinant HBV vaccines

The WHO guidelines on clinical investigation of HBV immunoglobulins recommends that new or significantly modified recombinant HBV vaccine formulations should have extensive product characterization, immunogenicity testing, safety testing and proof-of-concept studies in animals. Variations in manufacturing, alteration in vaccine formulation or change in the route of administration require immunogenicity studies together with adequate animal safety/toxicological studies. ${ }^{39,44}$ Pre-clinical investigation of $\mathrm{HBV}$ vaccines should follow WHO guidelines. ${ }^{39}$ As no effects, apart from those on immunity, are expected with sole HBV vaccines, safety pharmacological studies are also not required. Toxicology studies should be performed as per WHO guidelines. ${ }^{39}$ Such studies should also reflect the intended clinical use of the vaccine in special populations like neonates and children. The assessment of immune responses should rely on the anti-HBsAg antibody titer in serum, using a validated and standardized assay.

If a vaccine is proposed to contain a novel antigen dose and/ or an adjuvant, that should be studied in all target populations. New HBV vaccines are needed to be compared directly with at least one licensed vaccine for which there is sufficient experience. If there are substantial changes in manufacturing, the new vaccine should be compared to an approved existing one. New HBsAg vaccines ought to be initially tested in healthy adult volunteers. Once immunogenicity is demonstrated in adults, further research should be conducted in the younger target populations, as per the intended use. For clinical trials, enrolment should usually be restricted to subjects with a negative history of HBV vaccination or disease and also negative for HBsAg, anti-HBs, and anti-HBc. The dose of recombinant $\mathrm{HBsAg}$ requires justifications based on pre-clinical studies and, if necessary, formal dose-ranging studies in adults are needed. Studies in neonates may include those who are born to HBsAg-positive and/or -negative mothers based upon the study's objectives. Studies in infants may be limited to those born to HBsAg-negative mothers (with no birth dose of HBV immunoglobulin), with/without a prior birth dose of vaccine.

Studies should determine the proportion of seronegative individuals who achieve anti-HBs antibody titer $\geq 10 \mathrm{IU} / \mathrm{L}$ at approximately 4 weeks after completion of an initial course of $\mathrm{HBV}$ vaccination. For the comparison between a new vaccine and reference vaccines, the protocol and analysis should predefine a well-justified noninferiority margin ${ }^{45}$ to compare the proportion of individuals with $\geq 10 \mathrm{IU} / \mathrm{L}$ anti-HBs. The protocols should plan for secondary analysis of the proportion of subjects who achieve $\geq 100 \mathrm{IU} / \mathrm{L}$ anti-HBs and should present reverse cumulative distributions. ${ }^{46}$ Secondary analyses should also compare the geometric mean titer between vaccines, and studies may plan for formal comparison of geometric mean titer ratios.

Some of the clinical studies should be performed with different lots manufactured using the same process. A proper trial to demonstrate lot-to-lot consistency is not normally required unless any particular concern is present. ${ }^{44}$ Research may be conducted to assess immunogenicity, efficacy, and safety of new recombinant HBsAg-containing vaccines in populations at risk or not responding adequately to vaccination in view of the potential requirement for a higher antigen dose and/or adjuvant. The chance for possible immune interference between HBV vaccines and other routine coadministered vaccines needs to be investigated. The immunogenicity, efficacy, and safety of a new vaccine, when formulated with other components (combination vaccine), should be assessed.

Recently vaccines with a novel adjuvant are under development with the target of achieving better seroprotection rates. The conventional HBV vaccines used aluminium hydroxide as an adjuvant, whereas the new recombinant vaccine ('HEPLISAV-B') uses cytosine phosphoguanine oligonucleotide synthesized using bacterial DNA. This new recombinant vaccine was approved by the United States Food and Drug Administration in November 2017 after the trials demonstrated increased seroprotection rates over the conventional vaccines. ${ }^{47,48}$

\section{Strategies for improvement of the HBV vaccine}

Current $\mathrm{HBsAg}$ vaccines cannot elicit an adequate immune response in patients with chronic hepatitis $B$, as there are a high load of viral antigens in the circulation which will induce immunological tolerance. To circumvent this potential barrier, pre-S1 and pre-S2 polypeptides are used in the vaccine. The gene coding for HBV surface antigen consists of pre-S1, pre$\mathrm{S} 2$, and $\mathrm{S}$ regions. ${ }^{49}$ The main approach for improvement is to supplement $\mathrm{HBsAg}$ with the pre-S1 and pre-S2 portions of HBsAg. ${ }^{50}$ These polypeptides are present at a very low level in patients with chronic hepatitis B, as they are the important domains in the mature virions only. In addition, antibodies induced by the pre-S1 and pre-S2 region can prevent the entry of virions into the host hepatocytes. Therefore, pre-S1 and pre-S2 region can be used as the alternate strategies to improve the immunological response against HBV. ${ }^{51}$

The additional approaches include increasing the antigen dose, accelerated vaccination schedules, alternative (intradermal) vaccination route, use of new adjuvants like immunostimulatory DNA sequences, etc. ${ }^{14}$ Combined hepatitis $A$ and $B$ vaccination is an interesting tactic to enhance the heterologous immunogenicity. ${ }^{52}$ Additionally, immunostimulatory sequences are unmethylated CpG motifs with oligodeoxynucleotide sequences, which is one of the pathogen-associated molecular patterns (PAMPs) that will activate the Toll-like receptors present on the antigen presenting cells. This will subsequently activate the innate and adaptive immune responses. These immunostimulatory sequences 
Das S. et al: HBV vaccine and immunoglobulin: Key concepts

can be used as adjuvants in the HBV vaccine to elicit a strong immune response. ${ }^{53}$

\section{Therapeutic HBV vaccines}

The accomplishment of prophylactic HBV vaccination depends on neutralization of the invading HBV by antibodies, and eventually effective viral control. Instead, the key target of therapeutic vaccination is to induce a multifunctional and multispecific $T$ cell response against the major viral antigens as well as to activate humoral immunity. ${ }^{54}$ An ideal therapeutic vaccine would also induce neutralizing antibodies. However, it is controversial whether a therapeutic vaccine can achieve virus elimination or sustained viral control. ${ }^{54}$ For HBV, both innate and adaptive immunity against the virus should be triggered. Therapeutic vaccination against chronic HBV infection aims to overcome the immunosuppression induced by high viral load, tolerogenic liver environment, and T cell dysfunction. Therapeutic HBV vaccine based on protein or peptides against include administration of $\mathrm{HBsAg}$ with or without $\mathrm{HBCAg}$ (highly immunogenic) and vaccination with immune dominant HLA-A2-restricted HBcAg18-27 peptide epitope. The HBcAg18-27-Tapasin interaction leads to increased production of cytokine IFN- $\gamma$ and interleukin-2, it also enhances HBV-specific cytotoxic T lymphocytes which play a vital role in hepatitis B virus clearance. In animal models, the same peptide has shown enhancement of specific cytotoxic T Iymphocyte activity induced by the fusion protein-reduced HBV DNA and HBsAg levels, and decreased the expression of HBsAg and HBcAg in liver tissue of HBV transgenic mice. ${ }^{55}$

Genetic vaccination is also being tried with replication incompetent recombinant viral vector vaccines or with $\mathrm{HBV}$ DNA that are genetically engineered. Cell-based vaccine methods involve the transfer of peptide-containing antigenpresenting cells, autologous dendritic cells and transmission of functional HBV-specific CD $8^{+}$cells carrying HBV-specific $\mathrm{T}$ cell or chimeric antigen receptors. ${ }^{54}$

In the near future, therapeutic vaccines are expected to offer great relief in individuals living with chronic hepatitis $B$. Therapeutic vaccines in hepatitis offer a great platform for biomedical research. A study conducted by Zhao et al. ${ }^{56}$ in a mouse model to find the efficiency of pHBV vaccine in chronic hepatitis B showed that HBV vaccine decreased HBsAg and HBV DNA efficiently and safely in HBV carrier mice.

Researchers have also tried a combination vaccine, termed NASVAC, which contains HBsAg and HBcAg against pegylated interferon, in treatment-naive chronic hepatitis $B$ patients. At the end of 24 weeks, the high proportion of individuals with sustained control of HBV DNA was seen in the combination vaccine-treated group compared to the pegylated interferon group $(57.7 \%$ vs. $35 \% ; p<0.01) .{ }^{57}$ The GS- 4774 T cell vaccine designed to elicit hepatitis B virus (HBV)-specific T cell response was tried in a phase II trial, to study the safety, tolerability, and efficacy in chronic hepatitis $B$ patients. Though the vaccine was well tolerated, it failed to provide adequate clinical benefits. ${ }^{58} \mathrm{~A}$ trial conducted by Horiike et al. ${ }^{59}$ evaluated the efficacy of combination therapy of lamivudine and vaccine in patients with chronic hepatitis $B$. It is found that in a subgroup consisting of $\mathrm{HBeAg}^{+}$chronic hepatitis $\mathrm{B}$ patients who received lamivudine at a dose of $100 \mathrm{mg}$ daily for 12 months and also vaccine containing $20 \mu \mathrm{g}$ of HBsAG, intradermally, once every 2 weeks for 12 months, showed a higher proportion of individuals having a reduction in HBV DNA compared to those in the lamivudine alone group ( $100 \%$ vs. $48 \% ; p<0.05)$.

\section{Conclusions}

Immunization against HBV is safe and has been accepted worldwide as a part of the routine immunization program. Although recombinant HBV vaccines are the ones which are used prophylactically, HBV immunoglobulins are also used to offer immediate protection in certain circumstances. There are numerous factors which determine the nature and duration of protection for the HBV vaccination. Certain immunological and clinical phenomena guide the preclinical and clinical development process of HBV vaccines. Regulatory recommendations for such clinical development are specific and comprehensive. Upcoming approaches for improvement of HBV vaccination includes pre-S1 and preS2 portions of $\mathrm{HBsAg}$, increasing the antigen dose, accelerated vaccination schedules, alternative vaccination route, use of new adjuvants like immunostimulatory DNA sequences, etc. Therapeutic vaccination is being tried to activate humoral immunity and induce a multifunctional and multispecific T cell response, to counter the major HBV antigens for effective viral control.

\section{Conflict of interest}

The authors have no conflict of interests related to this publication.

\section{Author contributions}

Conceptualized the article and provided overall guidance (SS), and drafted the article (SD, KR, SKB, MG, and ASX).

\section{References}

[1] Luo Z, Li L, Ruan B. Impact of the implementation of a vaccination strategy on hepatitis B virus infections in China over a 20 -year period. Int J Infect Dis 2012;16:e82-e88. doi: 10.1016/j.ijid.2011.10.009.

[2] Meireles LC, Marinho RT, Van Damme P. Three decades of hepatitis B control with vaccination. World J Hepatol 2015;7:2127-2132. doi: 10.4254/wjh.v7. i18.2127.

[3] Kane MA. Global status of hepatitis B immunisation. Lancet 1996;348:696. doi: 10.1016/S0140-6736(05)65598-5.

[4] Lok AS, Zoulim F, Dusheiko G, Ghany MG. Hepatitis B cure: From discovery to regulatory approval. Hepatology 2017;66:1296-1313. doi: 10.1002/hep.29323.

[5] Habib S, Shaikh OS. Hepatitis B immune globulin. Drugs Today (Barc) 2007; 43:379-394. doi: 10.1358/dot.2007.43.6.1050792.

[6] World Health Organization. Hepatitis B. Available from: http://www.who. int/ith/vaccines/hepatitisB/en/.

[7] WHO Publication. Hepatitis B vaccines: WHO position paper-recommendations. Vaccine 2010;28:589-590. doi: 10.1016/j.vaccine.2009.10.110.

[8] World Health Organization. Recommendations to assure the quality, safety and efficacy of recombinant hepatitis B. vaccines. Available from: https: //www.who.int/biologicals/vaccines/TRS_978_Annex_4.pdf.

[9] Lee LY, Chan SM, Ong C, M Aw M, Wong F, Saw S, et al. Comparing monovalent and combination hepatitis $B$ vaccine outcomes in children delivered by mothers with chronic hepatitis B. J Paediatr Child Health 2019;55:327-332. doi: $10.1111 /$ jpc.14194.

[10] Greenberg DP, Wong VK, Partridge S, Howe BJ, Ward JI. Safety and immunogenicity of a combination diphtheria-tetanus toxoids-acellular pertussishepatitis $B$ vaccine administered at two, four and six months of age compared with monovalent hepatitis $B$ vaccine administered at birth, one month and six months of age. Pediatr Infect Dis J 2002;21:769-777. doi: 10.1097/01.inf.0000023959.66684.61.

[11] Schillie S, Vellozzi C, Reingold A, Harris A, Haber P, Ward JW, et al. Prevention of hepatitis B virus infection in the United States: Recommendations of the advisory committee on immunization practices. MMWR Recomm Rep 2018; 67:1-31. doi: 10.15585/mmwr.rr6701a1.

[12] Blumberg BS, Millman I, inventor; Institute for Cancer Research, assignee. Vaccine against viral hepatitis and process. United States patent US 3636191A. 1972 January 18. 
[13] Hepatitis B Foundation. History of hepatitis B vaccine. Available from: http: //www.hepb.org/prevention-and-diagnosis/vaccination/history-of-hepatitis-bvaccine.

[14] Leroux-Roels G. Old and new adjuvants for hepatitis B vaccines. Med Microbiol Immunol 2015;204:69-78. doi: 10.1007/s00430-014-0375-9.

[15] Zannolli R, Morgese G. Hepatitis B vaccine: current issues. Ann Pharmacother 1997;31:1059-1067. doi: 10.1177/106002809703100916.

[16] Leroux-Roels G, Cao T, De Knibber A, Meuleman P, Roobrouck A, Farhoudi A, et al. Prevention of hepatitis B infections: vaccination and its limitations. Acta Clin Belg 2001;56:209-219. doi: 10.1179/acb.2001.032.

[17] Lustbader ED, London WT, Blumberg BS. Study design for a hepatitis B vaccine trial. Proc Natl Acad Sci U S A 1976;73:955-959. doi: 10.1073/pnas.73.3. 955.

[18] van der Sande MA, Waight P, Mendy M, Rayco-Solon P, Hutt P, Fulford T, et al. Long-term protection against carriage of hepatitis $B$ virus after infant vaccination. J Infect Dis 2006;193:1528-1535. doi: 10.1086/503433.

[19] World Health Organization. Hepatitis B vaccines: WHO position paper, July 2017 - Recommendations. Vaccine 2019;37:223-225. doi: 10.1016/j. vaccine.2017.07.046

[20] Gesemann M, Scheiermann N. Quantification of hepatitis B vaccine-induced antibodies as a predictor of anti-HBs persistence. Vaccine 1995;13: 443-447. doi: 10.1016/0264-410X(94)00010-K.

[21] British Transplantation Society. Guidelines for hepatitis \& solid organ transplantation. Available from: http://bts.org.uk/wp-content/uploads/2018/03/BTS_ HepB_Guidelines_FINAL_09.03.18.pdf.

[22] Saltoğlu N, Inal AS, Tasova Y, Kandemir O. Comparison of the accelerated and classic vaccination schedules against Hepatitis B: three-week Hepatitis B vaccination schedule provides immediate and protective immunity. Ann Clin Microbiol Antimicrob 2003;2:10. doi: 10.1186/1476-0711-2-10.

[23] Rosman AS, Basu P, Galvin K, Lieber CS. Efficacy of a high and accelerated dose of hepatitis $B$ vaccine in alcoholic patients: a randomized clinical trial. Am J Med 1997;103:217-222. doi: 10.1016/S0002-9343(97)00132-0.

[24] Domínguez M, Bárcena R, García M, López-Sanroman A, Nuño J. Vaccination against hepatitis $B$ virus in cirrhotic patients on liver transplant waiting list. Liver Transpl 2000;6:440-442. doi: 10.1053/jlts.2000.8313.

[25] Ballinger AB, Clark ML. Severe acute hepatitis $B$ infection after vaccination. Lancet 1994;344:1292. doi: 10.1016/S0140-6736(94)90775-7.

[26] Yang S, Tian G, Cui Y, Ding C, Deng M, Yu C, et al. Factors influencing immunologic response to hepatitis $B$ vaccine in adults. Sci Rep $2016 ; 6: 27251$. doi: $10.1038 /$ srep27251.

[27] Tajiri K, Shimizu Y. Unsolved problems and future perspectives of hepatitis B virus vaccination. World J Gastroenterol 2015;21:7074-7083. doi: 10. 3748/wjg.v21.i23.7074.

[28] Purdy MA. Hepatitis B virus S gene escape mutants. Asian J Transfus Sci 2007;1:62-70. doi: 10.4103/0973-6247.33445.

[29] Hsu HY, Chang MH, Ni YH, Chen HL. Survey of hepatitis B surface variant infection in children 15 years after a nationwide vaccination programme in Taiwan. Gut 2004;53:1499-1503. doi: 10.1136/gut.2003.034223.

[30] Sticchi L, Caligiuri P, Cacciani R, Alicino C, Bruzzone B. Epidemiology of HBV S-gene mutants in the Liguria Region, Italy: Implications for surveillance and detection of new escape variants. Hum Vaccin Immunother 2013;9:568571. doi: 10.4161/hv.23236

[31] Bian T, Yan H, Shen L, Wang F, Zhang S, Cao Y, et al. Change in hepatitis B virus large surface antigen variant prevalence 13 years after implementation of a universal vaccination program in China. J Virol 2013;87:12196-12206. doi: 10.1128/JVI.02127-13.

[32] Tilzey AJ. Hepatitis B vaccine boosting: the debate continues. Lancet 1995; 345:1000-1001. doi: 10.5555/uri:pii:S0140673695907513.

[33] Lin AW, Wong KH. Long-term protection of neonatal hepatitis B vaccination in a 30-year cohort in Hong Kong. J Hepatol 2013;59:1363-1364. doi: 10. 1016/j.jhep.2013.08.021.

[34] Salisbury $D$, Ramsay $M$, Noakes K. Immunisation against infectious disease. Available from: https://webarchive.nationalarchives.gov.uk/20100330184831/ http://www.dh.gov.uk/prod_consum_dh/groups/dh_digitalassets/documents/ digitalasset/dh_113539.pdf.

[35] Are booster immunisations needed for lifelong hepatitis B immunity? European Consensus Group on Hepatitis B Immunity. Lancet 2000;355:561-565. doi: 10.1016/S0140-6736(99)07239-6.

[36] Geier MR, Geier DA, Zahalsky AC. A review of hepatitis B vaccination. Expert Opin Drug Saf 2003;2:113-122. doi: 10.1517/14740338.2.2.113.
[37] World Health Organization. The Global Advisory Committee on Vaccine Safety rejects association between Hepatitis $B$ vaccination and multiple sclerosis (MS). Available from: https://www.who.int/vaccine_safety/committee/ topics/hepatitisb/ms/en/.

[38] Sandhu P, Haque M, Humphries-Bickley T, Ravi S, Song J. Hepatitis B virus immunopathology, model systems, and current therapies. Front Immunol 2017;8:436. doi: 10.3389/fimmu.2017.00436.

[39] World Health Organization. WHO guidelines on nonclinical evaluation of vaccines. Available from: https://www.who.int/biologicals/publications/trs/areas/ vaccines/nonclinical_evaluation/ANNEX\%201Nonclinical.P31-63.pdf?ua=1.

[40] Han S. Clinical vaccine development. Clin Exp Vaccine Res 2015;4:46-53. doi: 10.7774/cevr.2015.4.1.46

[41] World Health Organization. Hepatitis B position paper. Available from: https: //www.who.int/immunization/policy/position_papers/hepatitis_b/en/.

[42] Gong J, Liu X. Effect of HBIG combined with hepatitis B vaccine on blocking HBV transmission between mother and infant and its effect on immune cells. Exp Ther Med 2018;15:919-923. doi: 10.3892/etm.2017.5474.

[43] European Medicines Agency. Guideline on the clinical investigation of hepatitis B immunoglobulins. Available from: https://www.ema.europa.eu/en/ documents/scientific-guideline/guideline-clinical-investigation-hepatitis-bimmunoglobulins_en.pdf.

[44] World Health Organization. Guidelines on clinical evaluation of vaccines: Regulatory expectations. Available from: https://www.who.int/biologicals/ Clinical_guidelines_revised_IK_29_Oct_2015.pdf.

[45] European Medicines Agency. Guideline on the choice of the non-inferiority margin. Available from: https://www.ema.europa.eu/en/documents/scientificguideline/guideline-choice-non-inferiority-margin_en.pdf.

[46] Reed GF, Meade BD, Steinhoff MC. The reverse cumulative distribution plot: a graphic method for exploratory analysis of antibody data. Pediatrics 1995; 96:600-603.

[47] HEPLISAV-B. Available from: https://www.fda.gov/media/108745/download.

[48] A two-dose hepatitis B vaccine for adults (Heplisav-B). Med Lett Drugs Ther 2018;60:17-18.

[49] Masuda M, Yuasa T, Yoshikura H. Effect of the preS1 RNA sequence on the efficiency of the hepatitis B virus preS2 and S protein translation. Virology 1990;174:320-324. doi: 10.1016/0042-6822(90)90083-4.

[50] Yum JS, Ahn BC, Jo HJ, Kim DY, Kim KH, Kim HS, et al. Use of pre-S proteincontaining hepatitis $B$ virus surface antigens and a powerful adjuvant to develop an immune therapy for chronic hepatitis B virus infection. Clin Vaccine Immunol 2012;19:120-127. doi: 10.1128/CVI.05355-11.

[51] Shouval D, Roggendorf H, Roggendorf M. Enhanced immune response to hepatitis $B$ vaccination through immunization with a Pre-S1/Pre-S2/S vaccine. Med Microbiol Immunol 2015;204:57-68. doi: $10.1007 /$ s00430-014-0374-x.

[52] Diepolder HM. Can specific heterologous immunity boost hepatitis $B$ vaccine responses? J Infect Dis 2008;198:297-298. doi: 10.1086/589721.

[53] Higgins D, Marshall JD, Traquina P, Van Nest G, Livingston BD. Immunostimulatory DNA as a vaccine adjuvant. Expert Rev Vaccines 2007;6:747-759. doi: $10.1586 / 14760584.6 .5 .747$

[54] Kutscher S, Bauer T, Dembek C, Sprinzl M, Protzer U. Design of therapeutic vaccines: hepatitis $B$ as an example. Microb Biotechnol 2012;5:270-282. doi: $10.1111 / \mathrm{j} .1751-7915.2011 .00303 . x$.

[55] Chen X, Tang Y, Zhang Y, Zhuo M, Tang Z, Yu Y, et al. Tapasin modification on the intracellular epitope HBcAg18-27 enhances HBV-specific CTL immune response and inhibits hepatitis B virus replication in vivo. Lab Invest 2014; 94:478-490. doi: 10.1038/labinvest.2014.6.

[56] Zhao HJ, Han QJ, Wang G, Lin A, Xu DQ, Wang YQ, et al. Poly I:C-based rHBVvac therapeutic vaccine eliminates HBV via generation of HBV-specific CD8+ effector memory T cells. Gut 2019. doi: 10.1136/gutjnl-2017-315588.

[57] Al Mahtab M, Akbar SMF, Aguilar JC, Guillen G, Penton E, Tuero A, et al. Treatment of chronic hepatitis $B$ naïve patients with a therapeutic vaccine containing $\mathrm{HBS}$ and $\mathrm{HBC}$ antigens (a randomized, open and treatment controlled phase III clinical trial). PLoS One 2018;13:e0201236. doi: 10. 1371/journal.pone.0201236.

[58] Lok AS, Pan CQ, Han SH, Trinh HN, Fessel WJ, Rodell T, et al. Randomized phase II study of GS-4774 as a therapeutic vaccine in virally suppressed patients with chronic hepatitis B. J Hepatol 2016;65:509-516. doi: 10 . 1016/j.jhep.2016.05.016.

[59] Horiike N, Fazle Akbar SM, Michitaka K, Joukou K, Yamamoto K, Kojima N, et al. In vivo immunization by vaccine therapy following virus suppression by lamivudine: a novel approach for treating patients with chronic hepatitis B. J Clin Virol 2005;32:156-161. doi: 10.1016/j.jcv.2004.07.004. 